\title{
NextGen Far-Term Concept Exploration for Integrated Gate-to-Gate Trajectory-Based Operations
}

\author{
Sally C. Johnson ${ }^{1}$ \\ Adaptive Aerospace Group, Inc., Hampton, VA 23666 \\ Bryan E. Barmore ${ }^{2}$ \\ NASA Langley Research Center, Hampton, VA 23681
}

\begin{abstract}
NASA is currently conducting concept exploration studies toward the definition of a far-term, gate-to-gate concept for Trajectory-Based Operations. This paper presents a basic architectural framework for the far-term concept and discusses some observations about implementation of trajectory-based operations in the National Airspace System. Within the concept, operators and service providers collaboratively negotiate aircraft trajectories, providing agile, optimized, aircraftspecific routing to meet service provider gate-to-gate flow-management constraints and increasing capacity by smoothly and effectively combining flight-deck-based and ground-based metering, merging, and spacing in a mixed-equipage environment. The far-term TBO concept is intended to influence the direction of mid-term TBO research and to inform the definition of stable requirements and standards for TBO communications infrastructure and user equipage.
\end{abstract}

\section{Introduction}

Trajectory-Based Operations (TBO) is one of the essential concepts in the NextGen transformation of the National Airspace System (NAS) ${ }^{1,2}$ and in the European Union's Single European Sky Air Traffic Management Research (SESAR) effort ${ }^{3}$. TBO is fundamental to achieving safe and efficient growth in global operations. Commercial air transport and regional jet traffic is expected to grow at a slow but steady rate for the foreseeable future; however, explosive growth is projected for Unmanned Aerial Systems (UAS) operating in the NAS, pushing the overall traffic demand far beyond the original predictions for 2025. If on-demand mobility ${ }^{4}$ succeeds as a business model, there may be a substantial increase in general aviation traffic, and many of these aircraft will be operating without experienced pilots onboard. Access to space and future supersonic transports must also be accommodated in future NAS operations. New environmentally friendly and clean-energy vehicles may require significantly different flight profiles to realize their environmental benefits, and there will likely be growing pressure to allow legacy aircraft to fly more fuel efficient flight profiles to reduce emissions. Thus, the future NAS will not only see substantial capacity increases, but also a large disparity in vehicle performance and equipage at all altitudes.

TBO utilizes 4-Dimensional Trajectories (4DTs) that span all phases of flight from pushback to arrival at the gate as the basis for planning and executing all flight operations. The mode of operations and the requirements of the airspace dictate the specificity of the trajectory. As the flight progresses, more detail is added to the downstream trajectory as needed for flow management, resource allocation, and separation assurance. Trajectories are negotiated between the operator and the Air Navigation Service Provider (ANSP), both preflight and during the flight as conditions change, to satisfy the operators' business objectives and preferences while meeting ANSP constraints. User preferences are accommodated to the greatest extent possible, and trajectories are constrained only to the extent required to accommodate demand or for other national concerns, such as safety, security, or environment. In highdensity or high-complexity airspace, the ANSP may need to limit the aircraft to a given published airway and assign constraints at specific points; while in low- to medium-density airspace, a wind-optimal route defined by a series of arbitrary points in space defined by latitude and longitude might be negotiated. The use of precise 4DTs dramatically reduces trajectory uncertainty and enables airspace to be used more effectively to safely accommodate high levels of demand, reduce environmental impacts, and maximize the use of capacity-limited airspace and airport resources. Furthermore, TBO will increase the predictability and stability of traffic flows, support a common operational picture

\footnotetext{
${ }^{1}$ Senior Research and Development Engineer, member.

${ }^{2}$ Aerospace Engineer, member.
} 
through use of electronic negotiation, facilitate more effective Collaborative Decision Making between airspace users and the ANSP, and enable increased levels of integrated automation across the NAS.

With TBO, integrated mixed-equipage operations are supported, and operators that equip their fleets to conduct TBO receive services from the ANSP that allow them to achieve operating benefits. Because TBO is a significant paradigm shift in the way flights are managed, the transition to TBO will occur gradually over time as flight-deck and ground-based automation is developed and as supporting infrastructure is implemented.

The FAA is committed to moving toward TBO and is making significant progress in working with EUROCONTROL to define and implement globally harmonized standards for key infrastructure to support the transition to TBO, such as Automatic Dependent Surveillance-Broadcast (ADS-B) In, Digital Data-Link Communications (DataComm), and System-Wide Information Management (SWIM). Near- and mid-term NextGen Collaborative Air Traffic Management (CATM) and traffic management tools, for use by either the ANSP or the airline dispatchers, as well as flight-deck based technologies are already moving towards some of the capabilities and benefits that full TBO is expected to provide. The FAA is developing a concept of operations for TBO and embarking on a demonstration in simulation of 4DT operations, including Dynamic Required Navigation Performance (D-RNP) and Advanced Interval Management (A-IM). D-RNP is a concept for rerouting of aircraft by the ANSP by uplinking a 4D trajectory, and A-IM entails extension of Interval Management (IM) to pair with other operations, such as along D-RNP routes.

NASA has been supporting the FAA in the development and implementation of TBO through research projects culminating in a series of Air traffic management Technology Demonstration (ATD) projects. As part of this effort, NASA has been conducting initial concept exploration studies leading toward defining a far-term, gate-to-gate TBO concept. The far-term TBO concept is intended to guide the direction of mid-term TBO research and to inform the definition of stable requirements and standards for TBO communications infrastructure and user equipage. This paper describes some observations from initial concept exploration studies.

\section{Concept Exploration}

Initial concept exploration studies have led to the definition of three pillars upon which NASA's far-term gate-togate TBO concept will be based:

1) Electronic collaborative trajectory negotiation

2) Agile, optimized, aircraft-specific trajectories

3) Integrated gate-to-gate flow management in a mixed equipage environment

Putting them together into a unified concept, operators and service providers collaboratively negotiate aircraft trajectories, providing agile, optimized, aircraft-specific routing to meet service provider gate-to-gate flowmanagement constraints, and increasing capacity by smoothly and effectively combining flight-deck-based and ground-based metering, merging, and spacing in a mixed-equipage environment. Each pillar is discussed in detail in the following subsections.

\section{A. Electronic Collaborative Trajectory Negotiation}

A key facet of TBO is ensuring that there are consistent views between all stakeholders and air and ground automation of how each trajectory will be flown, enabling more effective use of automation and providing predictability and stability in air traffic flow management as well as separation assurance.

\section{Trajectory Negotiation Process}

A detailed process for negotiation of aircraft trajectories to meet service provider constraints must be developed. The proper role for all of the agents needs to be determined. For short time horizon events, it is expected that the negotiations would occur between the air traffic controller and the flight crew. For longer time horizon changes or when many aircraft need updating simultaneously, the negotiations may start between the traffic manager and the Flight Operations Center (FOC) with the results being provided to the controller and flight crew for modification as necessary.

It is generally accepted that the ANSP has the best picture of traffic, national and regional weather impacts on traffic, and allocation of limited airspace and airport resources, such as runways; while the airborne/user domain has the best picture of the aircraft's flight performance characteristics, local weather conditions, and the user's business objectives. Thus, the process for negotiating trajectories will primarily involve the ANSP identifying a set of constraints on the flight, and the user defining a specific 4D trajectory, or a set of trajectory options, to meet the constraints, with iteration as necessary, adjusting 3D route or time. Some example constraints could include crossing 
times or altitudes at a point, conformance to a published navigation procedure, airspace to avoid, or ensuring a minimum distance relative to one or more other aircraft. A full set of possible constraints needs to be developed and agreed upon. The negotiation process should be reliable, highly automated, and converge quickly.

Preflight, trajectory negotiation will be conducted by the FOC, pilot, or UAS operator using ground-based tools with access to traffic flow information through System Wide Information Management (SWIM). The ANSP predicts the future state of NAS resources, estimating capacity and demand levels and anticipating areas of congestion that will require corrective action to mitigate. NAS traffic flow information is shared with aircraft operators through netcentric information sharing. When airspace constraints are expected, the FOC may submit one or multiple prioritized desired trajectories to the ANSP. These trajectories and associated data are known as a Trajectory Option Set (TOS). During a flight, trajectory negotiation will sometimes be performed by pilots onboard using an Electronic Flight Bag (EFB) or trajectory negotiation capability integrated with their avionics systems, while others will rely on an FOC for renegotiation or accept trajectory clearances from the ANSP without negotiation. For aircraft equipped for electronic negotiation of trajectories, minor tactical deviations would be handled by the pilot and ANSP, while the FOC may be involved if constraints must be renegotiated and fleet management decisions are involved.

The agreed-upon trajectory would include tolerances in all relevant dimensions (lateral, altitude, speed, timing, etc.). This means that the $4 \mathrm{D}$ trajectory is really a $4 \mathrm{D}$ volume within which the aircraft will be. Where necessary, these tolerances can be small. For example, the aircraft may need to be within 0.1 nautical mile and 5 seconds of the agreed upon location to support parallel runway operations. When practical, the tolerances should be large enough to limit the need to renegotiate a trajectory. For example, in en route airspace, a metering time may only need to be met within one minute, allowing the aircraft to more efficiently meet other constraints or optimize their behavior.

Trajectory negotiation proceeds through phases of pre-negotiation, negotiation, agreement, and execution ${ }^{2}$. During the pre-negotiation phase, information relevant to negotiating the trajectory is gathered by the initiator, such as determining a conflict-free route or estimating the arrival time at a downstream point. During the flight, trajectory renegotiation can be initiated by the ANSP for a number of reasons, including separation management, flow management, or to improve flight efficiency. The FOC might negotiate a change in a downstream constraint as part of fleet management, such as the time window for entering capacity-constrained en route or terminal area airspace or negotiation of a more efficient route. The pilot might initiate a trajectory change for many reasons, such as a minor deviation around convective weather, an altitude change to avoid turbulence, to request a more optimized route, or upon recognizing that the aircraft is no longer able to meet the current trajectory due to unexpected conditions.

The negotiation phase depends in part on how the negotiation is initiated. If new constraints have been generated by the ANSP, the airborne or ground-based user system would ingest these constraints and determine a trajectory that would satisfy the constraints while also complying with the aircraft's performance capabilities and the user's business objectives. This proposed optimal trajectory would be communicated to the ground domain where it would be either accepted or a new or modified set of constraints would be sent back to the user system. Ideally, this negotiation would take one or two cycles before agreement is reached. Likewise, if the user initiates the negotiation, the ANSP receives the trajectory or TOS from the FOC/user via automation and the trajectory options are evaluated in the context of aggregation of large numbers of trajectories. The automation selects the highest-priority trajectory that is feasible based on NAS constraints, and the ANSP transmits the trajectory option selected to the FOC/user. If none of the trajectory options are feasible, or as needed as the flight progresses, the FOC/user may develop and submit a modified TOS to better align with their objectives. In such a case, the ANSP once again will consider the desired trajectories and transmit a new acceptable selection. The trajectory negotiation process must be performed in a timely manner, especially for inflight trajectory re-negotiation.

A significant research question is how to enable rapid negotiations, likely requiring highly automated systems, while allowing for appropriate human setting of goals and acceptance of the negotiated solution. When time-critical constraints require the immediate action of the controller (e.g., to ensure safe separation is maintained), the negotiation phase will be skipped and the controller then proceeds immediately to the agreement phase. When immediate action is required by the aircraft to insure safe operation is maintained (e.g., Traffic Collision Avoidance System (TCAS) resolution advisory), the trajectory renegotiation, if required, is initiated by the pilot after the immediate safety issue has been resolved. In a mature TBO environment, it is expected that such tactical reactions would be infrequent occurrences.

The agreement phase is very brief and consists of the issuance by the ANSP and acceptance by the flight crew of the clearance that represents the negotiated trajectory. The agreed-upon trajectory would be executed, with the user and the ANSP both independently monitoring for trajectory conformance. During the flight, each party would reevaluate the situation periodically to determine if the initial constraints and agreed-upon trajectory are still satisfactory. Either party could initiate a new negotiation, with the ANSP adding, changing, or eliminating constraints, or the operator proposing a new trajectory to meet the existing constraints. 


\section{Trajectory Data and Format}

NASA plans to conduct extensive research aimed at defining the air/ground and ground/ground information exchange requirements to support effective TBO operations for each phase of flight. This research will consider the types of operations to be supported and the trajectory prediction accuracy needed for various levels of performance of the operations, attempting to balance the need for more accuracy against the communication bandwidth and processing required. Using a performance-based approach, aircraft flying in specific airspace or conducting specific operations might be required to transmit highly accurate trajectory information periodically or upon significant changes in trajectory.

Most civil transport and high-end business general aviation aircraft are equipped with a Flight Management System (FMS) that will deliver the aircraft along the trajectory with a reasonably high level of accuracy and predictability. The trajectory that the FMS is attempting to fly is often referred to as the reference trajectory, since it is used as the reference for the aircraft's guidance system. A number of inaccuracies are introduced as the aircraft's guidance system attempts to fly the reference trajectory, such as sensor errors, navigation errors, and anomalies due to the guidance strategy used by the FMS, which are particularly pronounced in climbs and descents. Since different FMSs fly trajectories differently, identification of future waypoints alone is insufficient for safely and efficiently managing air traffic.

Today's NAS ground-based decision-support tools predict how a waypoint-based trajectory will be flown by each aircraft using models of aircraft performance combined with complex algorithms that account for meteorological conditions, predicted user preferences, and anticipated ANSP actions. Thus, they are not trying to predict the reference trajectory, or the actual trajectory that would result from the FMS attempting to fly the reference trajectory. Instead, they are also trying to correct the trajectories for anticipated ANSP and pilot actions. For example, since the aircraft are not being managed by trajectories today but manually by tactical instructions such as vectors or speed instructions, many ground-based tools anticipate what speed and other clearances will be given to the aircraft by the current, upstream, or downstream controller based on procedures and norms specific to the local airspace. We will refer to this as the corrected trajectory. Each ground-based tool is developed independently and then adapted to each deployment site and may predict trajectories using different ANSP/pilot assumptions as well as different models of aircraft behavior and equations of motion, which is a major impediment to integration and modification of today's groundbased support automation ${ }^{5}$. It is important to note that the developers of ground-based tools are not adding in these assumptions in order to unnecessarily complicate the decision-support automation. These built-in assumptions are tailoring the tools to the specific needs of the controller, enabling the tools to be used more effectively by the controller in managing the traffic.

Recently, particularly in Europe, the research focus has shifted from trajectory prediction to trajectory synchronization, where detailed trajectory information is downlinked from the FMS to ensure that the ground-based automation has an accurate view of how the trajectory will be flown by the FMS. There has been important work in creating languages and notations for clearly capturing and communicating an aircraft's trajectory, including the Aircraft Intent Description Language (AIDL) developed by Boeing Research and Technology Europe, which "completely and unequivocally" describes how the FMS will implement the trajectory ${ }^{6}$, but also requires significant communication bandwidth to downlink.

The FAA has been working cooperatively with EUROCONTROL and the International Civil Aviation Organization (ICAO) to define a common framework for communication of trajectory information. The near-term goal of the trajectory downlink is to correct for errors in flight plan trajectory information due to tactical maneuvering of the aircraft for separation assurance or weather deviations, but the trajectory downlink may potentially support TBO's needs for trajectory communication and negotiation. Controllers today are not incentivized to consistently update flight plans to account for tactical maneuvering of the aircraft for separation assurance or weather, and FAA studies have shown that lateral errors of 20 to $30 \mathrm{NM}$ are common in the NAS and in Europe, and that these lateral errors in intent information result in poor performance of conflict detection tools 7 .

The ICAO planning framework includes 4D Trajectory Data-Link (4D-TRAD), where the 4D trajectory is downlinked from the aircraft's FMS, negotiated if necessary, agreed through the uplink of a clearance, and subsequently flown by the aircraft. RTCA and EUROCAE developed a joint standard for trajectory downlink called Extended Projected Profile (EPP). The EPP message is a negotiated subset of full trajectory information, transmitted via Automatic Dependent Surveillance - Contract (ADS-C). NASA intends to conduct analyses and simulation studies to determine whether the data fields contained in the EPP message are sufficient for supporting far-term TBO. If, for example, a minor addition to the EPP message set would result in significant improvement in future TBO performance, NASA could recommend this addition as part of defining a future version of the EPP standard.

A joint study using trajectory downlink and flight information from previous flight studies was conducted by Airservices Australia, FAA, EUROCONTROL, GE Aviation Systems, and Boeing Research \& Technology Europe 
to evaluate the use of the EPP message for trajectory synchronization as required for EUROCONTROL decisionsupport automation ${ }^{8}$. In this study, it was shown that even combining the EPP downlink with accurate aircraft performance models provided by the Base of Aircraft Data Family 4 (BADA4) ${ }^{9}$, and the inclusion of aircraft mass in the EPP, was not sufficient for the trajectory synchronization in climb and descent. Laterally, the EPP message appeared to be sufficient, with the exception of fly-over waypoints, since the radius of fly-over waypoints is not included in the message. In climb and descent, aircraft performance characteristics, including how the FMS performs the climb and descent plus unknown drag components lead to significant errors in trajectory prediction; however, they have developed and demonstrated a method for using the EPP downlink to calibrate the trajectory prediction process, with accuracy sufficient for trajectory synchronization as required for EUROCONTROL decision-support automation. They concluded that although the current EPP definition does not include all variables that impact the air-ground trajectory synchronization process, with a single EPP downlink, the trajectory prediction process can be synchronized through a calibration function, ensuring prediction of high accuracy 'what if' trajectories and thereby anticipating the FMS behavior upon changes in aircraft intent. A similar analysis will need to be conducted to evaluate the suitability of the EPP message to support trajectory synchronization for U.S. decision-support automation for TBO. This analysis is again complicated by the plethora of U.S. ground-based tools and divergence of trajectory prediction algorithms.

If the EPP message thus solves the problem of trajectory synchronization for suitably equipped aircraft, there is still the problem of needing to build assumptions into the trajectory predictions to generate corrected trajectories, tailored to the performance and usability of ground-based automation. There seem to be two general approaches to solving this issue. One solution, building on a proposal by a team from Airservices Australia, Boeing Research and Technology Europe, and Universidad Politécnica de Madrid ${ }^{10}$, is a two-stage approach to ground-based trajectory prediction. The first stage is to compute the reference trajectory that is held by the FMS, and the second stage is to compute from the reference trajectory the actual trajectory that will be flown by the aircraft. In the first stage, the reference trajectory would by based on the EPP message, and the EPP message format would be used for sharing of trajectory information among all ground-based automation via SWIM. A predicted trajectory would still be generated for aircraft that are not equipped for or participating in trajectory downlink. In the second stage, the actual trajectory would be generated from the reference trajectory, ideally with common assumptions and models used between tools. The corrected trajectory could then be generated as an additional third stage. The separation of predicting the actual trajectory and then adding the correction assumptions would be beneficial in standardizing tools, facilitating integration and enabling intercommunication between tools.

A second solution that deserves study is the continual refinement and downlink of trajectory information by the FMS during the flight instead of correcting the trajectory on the ground, in essence closing the loop on the trajectory. If a correction is necessary, rather than correcting the trajectory on the ground, a corrected trajectory is negotiated and constraints applied. If the aircraft is still able to meet all assigned constraints, the trajectory might not have to be renegotiated, but just updated via downlink from the FMS. For example, if the aircraft is assigned a time window to arrive at a given point, the FMS would periodically report the Estimated Time of Arrival (ETA) for arriving at the point as part of the EPP message. Likewise, if the aircraft is performing IM, keeping appropriate spacing behind another aircraft, the waypoint ETAs in the EPP message give the timing information needed by the ground-based automation. Instead of building models of controller behavior into the tools, the approach would be to build that behavior into the trajectories and consistently updating the trajectories as needed. In a TBO environment, the ANSP must have decision-support tools and procedures that make it easy and automatic to update the trajectory information as a strategic or tactical maneuver is communicated to the aircraft, keeping the trajectory synchronized.

\section{B. Agile, Optimized, Aircraft-Specific Trajectories}

Trajectory negotiation is a balance between optimization of individual flights and global optimization of traffic flows to maximize throughput. A series of waypoints will be used to identify a route optimized based on winds, weather, user preferences, and other factors. Users have different preferred trajectories for a number of reasons, including business objectives (time vs. fuel) and willingness to traverse conditions such as moderate convective weather and atmospheric turbulence. Where feasible, trajectories should be tailored to the aircraft performance and optimized to meet the business objectives of the user, and the trajectory negotiation process should be agile enough to allow re-optimization of trajectories as conditions change throughout the flight.

For aircraft equipped with DataComm, the waypoints can include arbitrary points defined by latitude/longitude; however, aircraft not equipped with digital DataComm will receive clearances via voice communications and will be restricted to named waypoints. In high-density airspace, trajectories may be constrained to airways or specific offsets from airways that are dynamically defined by the ANSP. In medium- to low-density airspace, many trajectories will likely be independent of the airways system, but will still conform to controller conventions, such as eastbound/westbound altitudes. Fuel-efficient Optimized Profile Climbs (OPCs) and Continuous Descent Approaches 
(CDAs) should be supported routinely for all aircraft, even in the presence of high traffic demand and operational disruptions.

All aircraft managed by ANSPs (on Instrument Flight Rules (IFR) flight plans), including UASs, will be managed by trajectory. Preflight, trajectories will likely be negotiated by the FOC or filed by the user using ANSP-provided tools for CATM with access to NAS information such as Special Use Airspace status and potential future Traffic Management Initiatives (TMIs). Some pilots will have onboard capabilities for inflight trajectory renegotiation using an EFB or integrated with their avionics systems, while others will rely on an FOC for renegotiation or accepting trajectory clearances from the ANSP without negotiation. UAS operators will have the capability for trajectory negotiation, and some UAS will renegotiate trajectories in flight using onboard automation. Research will be needed to integrate loitering UAS in the NAS, including an effective means for communicating their loitering trajectories. If On-Demand Mobility succeeds as a business case and is introduced in the NAS, trajectory negotiation for those aircraft without experienced pilots on board will likely be accomplished by FOCs or onboard automation.

The 4DT for a flight covers the entire flight from pushback to arrival at the gate; however, it typically only includes specific detail for the part of the flight that is imminent, with more specificity for each phase of flight added as the flight progresses. If the flight is impacted by ANSP constraints, a 4DT will be negotiated with enough detail to accommodate the constraints. For example, during preflight planning many days in advance, the operator typically proposes a flight plan with basic information such as take-off time, origin, and destination. As the day and time for the flight approaches, the operator will be notified if the flight is projected to be affected by one or more constraints due to weather, excessive demand, etc., and in response, the operator will negotiate the timing and flight path, defined as a set of waypoints. If the destination airport or en route airspace is capacity-constrained, the flight may be subject to Time of Arrival Control (TOAC), with an assigned window for arriving at a waypoint just prior to the constrained region. The takeoff time for the flight will be calculated based on arriving within the TOAC window, and a conflictfree taxi route and timing will be calculated to deliver the aircraft to a short runway queue just before the takeoff time. Once the aircraft takes off and completes climb-out, the FMS can accurately calculate the aircraft's timing performance over the cruise portion of the flight, and this highly accurate 4DT information is shared with the ANSP. Thus, although flights are managed via 4DTs from preflight planning through all phases of flight, TBO does not place unnecessary constraints on the flight, but rather provides for flexibility to deal effectively with uncertainties in the system, such as weather.

Once the flight commences, the FMS on-board an aircraft performs two key functions for TBO. First, the FMS constructs the planned trajectory based on route information provided by the flight crew, information in the navigation database, atmospheric forecast information, and aircraft performance and state data. The state data includes items such as the current position and velocity as well as weight and expected fuel flow, among other variables. The second TBO function provided by the FMS is providing flight guidance to the aircraft control systems to fly along the planned trajectory. The FMS will attempt to optimize the planned trajectory based on the cost index provided by the flight crew. The optimized trajectory is limited by several constraints applied either by the flight crew or by the use of established procedures from the navigation database. The details of this highly accurate trajectory will be transmitted to ground automation and made available across the system via SWIM. Whenever downlinked accurate trajectory information is available for a flight, that information should be used by ground automation in place of independent trajectory prediction.

\section{Automation Support for Separation Assurance}

Controllers currently separate and manage air traffic manually with limited decision support automation, using radar screens to display streams of traffic on predefined airways and making cognitive operational judgments to ensure safe and efficient traffic flows. Controllers become experts at efficiently handling the specific flow patterns within their sectors, quickly examining streams of traffic to ensure safe spacing and managing the interleaving of traffic at intersections and merge points. Controllers are responsible for safely separating the traffic even when their screens freeze, which happens on rare occasions. Since the intensive mental processes of the controller are based on airspacespecific scan patterns, extensive improvements in decision-support automation for ANSPs are required to enable the widespread use of agile, optimized, aircraft-specific trajectories.

If a controller is responsible for manually separating traffic when tools fail him, then it is likely that most traffic in high-density, high-complexity airspace will be constrained to published airways. State of the art automated conflict detection and resolution systems are not yet reliable enough for controllers to be able to depend on them for decision support. Historically, one of the main roadblocks to automated conflict detection and resolution is the lack of accurate trajectory intent information. The use of downlinked ADS-C EPP messages to synchronize trajectory intent information holds significant promise in improving the performance of decision-support automation for conflict detection and resolution as well as for management of flows of traffic. The use of EPP information combined with 
ADS-B surveillance and airborne and ground-based conformance monitoring may provide enough data to support fully automated conflict detection and resolution in airspace where all aircraft are equipped with DataComm and transmitting updated EPP messages as conditions change. The fully automated separation assurance algorithms could be implemented in ground-based automation or in an airborne system, with intent information provided via Aircraft Access to SWIM (AAtS). With fully automated separation management, the need to keep aircraft on published airways likely goes away, since computer algorithms do not rely on the pattern-matching techniques used in human monitoring of traffic separation. Partial or full automation of separation assurance is a paradigm shift that will be extremely difficult to accomplish. The importance of reliability in decision-support automation cannot be overemphasized. Today's air traffic control process is quite mentally intensive, and the controller has no margin for second-guessing tools that might be giving bad information. For more than a decade, the Formal Methods group at NASA has been conducting research in the application of safety-critical system verification techniques to air traffic management applications, and they have extensive experience in the development and formal verification of separation assurance systems ${ }^{11,12}$. Formal Methods is the application of formal mathematical proofs to verify that an algorithm, design, or implementation is correct for all possible inputs, and they have been applied successfully in a number of applications by Microsoft, Rockwell Collins, Airbus, and others. The group is currently working on a formal definition of trajectories and their associated properties ${ }^{13}$. There are characteristics of the TBO domain that make this verification approach difficult, such as complex calculations using floating-point arithmetic and the use of heuristic algorithms, but the group has recently proposed a software validation technique that is directly applicable to TBO computations. Establishing the correctness and hence safety of the algorithms that implement critical functions of TBO is fundamental to establishing the safety of a future TBO system and its operational concept. A verification of this scope and criticality will require a break-through in software verification.

Conformance monitoring is an important aspect of TBO. If tactical and strategic management of flights is to be performed through trajectories, then it must be assured that aircraft are conforming to their agreed-upon trajectories, and conformance monitoring should be performed both on the flight deck and by ANSP automation. An aircraft that is not conforming to its agreed-upon trajectory must be treated differently because its future behavior is uncertain.

TBO automation and procedures must be interoperable with current and future collision avoidance systems. Before new operational concepts are implemented, there must be an analysis to ensure that there is a sufficiently low probability that the new operations will cause false alerts to occur. When a Resolution Advisory (RA) is received from TCAS or Airborne Collision Avoidance System (ACAS), or a safety alert is received from the ANSP ("traffic alert" via voice), the pilot is responsible for following the guidance given by the automation or the voice command. Once the pilot has conducted the maneuver to avoid the perceived imminent collision, the pilot then communicates with the ANSP for further guidance. The maneuver may be minor enough to not cause a measureable change in the aircraft's trajectory or to impact pairwise operations such as IM; however, a process must be defined for ensuring that the trajectory is updated as needed and that impacted operations are handled safely.

\section{The Effects of Weather on the NAS}

Weather can cause huge disruptions in the NAS for two reasons: there is significant uncertainty associated with weather prediction, and weather can stretch across hundreds of miles, causing NAS-wide impacts. While current efforts to improve weather information-gathering and prediction methods and to share weather information with all stakeholders through SWIM will improve the flight planning process, weather predictions will remain uncertain in terms of when, where, and how severe the weather conditions will evolve.

Widespread severe weather obviously can be extremely disruptive to NAS operations, but even localized weather issues pose challenges for capacity and efficiency. The presence of low ceilings significantly impacts arrival and departure rates at airports, and TBO has the potential to mitigate some of this impact through IM and Closely Spaced Parallel Runway operations. Localized severe convective weather can block arriving and departing or en route streams of traffic, and D-RNP routes have been proposed for deviating streams of traffic around weather. The ANSP and FOC probably have better global weather information than the aircraft, but this information could be sent to the cockpit via AAtS. The aircraft typically has a better picture of local weather requiring minor deviations.

NASA has conducted a scenario-based analysis of the effects of weather on the implementation of TBO concepts, such as D-RNP and A-IM, and a more in-depth study of these concepts in the presence of detailed realistic weather data is planned. NASA has extensive research experience developing and evaluating tools that continuously search for opportunities to improve the efficiency of trajectories around weather, including the airborne tool Traffic Aware Strategic Aircrew Requests (TASAR) ${ }^{14}$, the FOC tool Dynamic Weather Routes (DWR) ${ }^{15}$, and the ANSP tool Dynamic Reroutes for Arrivals in Weather (DRAW), formerly called Dynamic Arrival Routes (DAR) ${ }^{16}$. From experience gained from operational assessments of DWR, it is clear that predictions made more than two hours into 
the future of airways and arrival routes that will be impacted by severe weather at the time a flight would use them are highly uncertain; and there are noticeable uncertainties even 30 minutes out. In fact, DRAW frequently identifies that a flight approaching the terminal area that was rerouted preflight onto an inefficient arrival route because its original efficient arrival route is forecasted to be blocked, can now be rerouted back to its original arrival route. This points to the need for agility in the system to respond in real time with trajectory renegotiation as the weather evolves. The key weather-related research questions for implementation of trajectory-based operations, such as D-RNP and AIM, are the following:

- In the presence of moving weather cells, would a D-RNP route through a gap in the weather persist long enough for a stream of aircraft to traverse it or is aircraft-specific routing required?

- Which is more efficient for flow of traffic through a gap in the weather, a "gaggle" of aircraft on aircraftspecific routes or a "formation" flight of aircraft on D-RNP routes ${ }^{17}$ ?

- Would D-RNP routes for deviations around weather typically be short enough to be contained within a single center, or would they be long routes with coordination across multiple centers?

- Could/should en route D-RNP routes be predefined, or created in real time by ANSPs?

- Could A-IM or perhaps delegation of separation assurance responsibility to the flight deck for multiple aircraft picking their way through a gap in the weather increase the throughput of aircraft through the gap and alleviate controller workload for managing the aircraft shooting the gap?

- For arrival/departure, can an A-IM capability be developed that would allow continuation of IM operations even if one or both aircraft need to make a minor deviation for weather within a predefined corridor. How would the relative distances of the trajectories be calculated and communicated? Would an EPP message give enough information to support this? What if the target aircraft is not DataComm equipped and is not transmitting an EPP message?

\section{Gate-to-Gate Integrated Flow Management}

The Air Traffic Control System Command Center (ATCSCC) oversees the management of the timing and routing of flights before they depart, with a look-ahead horizon of two to 15 hours, focusing on ensuring that demand will not exceed capacity in any sectors or terminal areas across the NAS. Recent improvements in strategic traffic flow management include extending the support for digital dissemination of information to stakeholders. The Traffic Flow Management System (TFMS) now includes an interface system providing operators and other NAS stakeholders with information on the state of the NAS and current and potential future TMIs. NAS information now available to operators includes predictions of air traffic demand for the next fifteen hours, identification of airports, sectors, and fixes where excess demand is forecast, weather information, Flow-Evaluation Areas (FEAs), which are volumes of airspace that are expected to require TMIs, and reroutes that have been issued by the ATCSCC. The Collaborative Trajectory Options Program (CTOP) supports Collaborative Decision Making, enabling operators to respond to potential future TMIs by proposing potential plans for cancelling and rerouting impacted flights, including submission of multiple prioritized alternative trajectories for each affected flight.

In anticipation of the transition to TBO and moving from Collaborative Decision Making to more extensive CATM, a number of research studies have proposed traffic flow planning strategies that are more robust to weather uncertainties $^{18,19}$, and NASA is building upon these ideas. While effective preflight traffic flow planning that is robust to weather uncertainties is important, there is also a great need for responding with agility in real time as the weather evolves by flexibly managing flows to match capacity changes and renegotiating more efficient trajectories as they become available. Flexibly managing flows will likely require passing of capacity parameters and flow information between ground-based tools across sector and center boundaries rather than the manual flow-rate parameter settings used in today's tools. Renegotiating aircraft-specific trajectories to effectively meet user business objectives as weather conditions change will require new automation and procedures. Idris et al have proposed a concept where users take a more active role in traffic flow planning, with collaboration and responsibility shifting between FOC and ATSP dynamically as time-to-flow-constraint changes ${ }^{20}$.

Geographically dispersed Traffic Management Units (TMUs) manage local en route and terminal area traffic flow. Disruptions, such as hazardous weather or reduced throughput due to Instrument Meteorological Conditions (IMC), are handled manually by moving groups of aircraft, with limited negotiation with FOCs to prioritize flights. Measureable improvements in management of terminal-area traffic flows have been demonstrated by the deployment of Time-Based Flow Management (TBFM) ${ }^{21}$, based on NASA's Traffic Management Advisor (TMA) ${ }^{22}$, which conditions the flow of arrivals into a busy airport. TBFM predicts the trajectory of each aircraft along its projected 3D arrival, detects spacing conflicts, and generates information to support the controller in handling the spacing issue. The scheduled times of arrival of each aircraft are allowed to change until they cross the freeze horizon (an arc prior 
to the top of descent), at which time the aircraft's sequence and scheduled times of arrival are frozen. NASA has been working with the FAA to investigate the integration of IM operations with TBFM to support both ground-based and flight-deck-based merging and spacing of aircraft on arrival ${ }^{23}$, 24 . These near-term improvements are only precursors to TBO, and current operations are far below the traffic throughput and efficiency benefits to be gained from the transition to gate-to-gate TBO. Site adaptation issues and performance limitations of these improvements ${ }^{25}$ have provided valuable evidence of the importance of a system-wide approach to flow management with integration of ground-based tools across sectors and the need for automated system-wide optimization rather than use of manual parameter settings for each site.

A quote from a European project leader on Avignor's Arrival MANagement (AMAN) tool highlights the difficulty of developing useful decision support automation: "A controller would like an AMAN to be stable, correct, and strict when traffic builds up - and a flexible, mind-reading, and dynamic tool in other periods ${ }^{26}$." When traffic is light, the controller needs flexibility to tailor procedures for individual aircraft to enable fuel-efficient and time-saving flight operations. When traffic is heavy, the controller needs to be able to confidently depend on the tool. Today's deviations for weather are typically conducted in an ad hoc fashion, with the controller frequently transmitting tactical clearances by voice without updating flight plans. Controllers don't see the need and don't feel that they have the time to modify flight plans in these situations. If flight plans (trajectories) are not updated, then scheduling tools, such as TBFM, cannot adjust the times appropriately and thus cannot be used at the time that they are most needed to manage the flow of aircraft into a capacity-constrained airport. For TBO to work effectively, there must be consistent use of decisionsupport automation throughout the NAS, and the various tools must exchange accurate information unambiguously. This will only happen if tools are developed with the flexibility to effectively support all types of operations, so that controllers find them easy to use and helpful in all traffic situations. Only then can training be successful in modifying controller behavior to ensure that a trajectory modification is entered into the automation before giving a tactical clearance to the pilot unless it is truly an emergency.

Support for aircraft-specific routing will require significant improvements in ground-based automation to efficiently handle routing and rerouting without adversely impacting workload for the ANSP. Operational trials of the DWR system conducted at American Airline's Operations Center in Fort Worth, TX, showed that although DWR was able to identify a high number of potential time-saving reroutes near areas of convective weather, only eleven percent of DWR's potential route time savings were actually realized operationally, primarily due to ANSP staffing limitations ${ }^{27}$. FOC and ANSP workload will be significant issues in aircraft-specific routing, and clearly this process must be supported with decision support automation, not only to create optimal routes and reroutes, but also to communicate and negotiate these aircraft-specific reroutes without significantly increasing workload.

NASA is exploring the use of a new generation of TBO airborne tools and procedures for metering, merging, and spacing to enable aircraft to fly individualized trajectories while offloading controller workload. Sector capacity limits today are based on how many aircraft a controller can handle at once within a sector, but only if most of the aircraft are on published airways, with the controller focusing attention on the spacing within the streams of traffic moving along the airways and on the interleaving of traffic at intersections. If a controller had suitable automation support to enable the delegation of pairwise metering, merging, or spacing operations to aircraft that are operating off of published airways when intersecting with streams of traffic or merging onto published airways, this could perhaps alleviate some of the burden of monitoring those aircraft.

There have been a number of flight trials to explore the use of RTAs for trajectory management and TOAC. Studies in 2001-2008 showed that existing RTA technology can reliably predict and control to a trajectory in en route and terminal airspace ${ }^{28,29,30,31}$. The FAA conducted two sets of RTA flight trials in 2010 and $2011^{32}$, demonstrating that aircraft can meet desired RTA time performance to deliver aircraft to the Scheduled Time of Arrival assigned by TMA, but there were a number of operational issues uncovered in implementing TOAC in the NAS. Only $71 \%$ of the flights that were assigned an RTA fully executed the RTA within time tolerance to the meter fix, with $16 \%$ cancelled by the controller. Operational issues included controller and pilot concerns with RTA speed profiles, pilot workload in managing speeds and auto-throttles, controller workload in managing spacing in heavy traffic, and the "fragility of RTA under current operating conditions." In Europe, flight demonstrations were performed in 2013 to demonstrate the Initial 4D trajectory management concept developed under SESAR's TimeBased Operations concept, demonstrating the use of uplinked RTAs for TOAC and downlinked EPP messages for trajectory synchronization between stakeholders ${ }^{33}$. While all of these studies point to the likely eventual operational and technical feasibility of TOAC operations for managing flow, a number of operational issues are commonly reported that must be resolved, including controllers feeling the need to maneuver other aircraft out of the way to clear the way for the test aircraft. NASA's research in implementation of TOAC will build on the lessons learned from these previous studies. 
Gate-to-gate flow management is a complex mix of local and global optimization. The flow within a local area of high-density airspace must be optimized, and the flow into the high-density area needs to be managed to ensure safe and efficient flights. Our concept includes the following characteristics:

- Smoothly and effectively combining ground-based and airborne metering, merging, and spacing techniques.

- Continual use of automation and optimization, even in the presence of high traffic demand and disruptions, such as missed approaches and areas of hazardous weather that must be avoided.

- Ground-based integrated arrival/departure/transition/surface decision-support tools supporting CATM.

- En route Miles-In-Trail replaced by regional metering to manage airspace capacity and condition arrival flow; where possible providing alternative routes or passing lanes to allow for fleet optimization where aircraft are headed to the same destination airport and for optimization of individual flights where aircraft are not subject to the same downstream constraints.

- En route controller workload alleviated by use of TOAC and flight-deck-based spacing where prolonged relative positioning is an issue.

- CDAs and OPCs for all aircraft in streams of mixed-equipage traffic enabled by effective combination of flight-deck-based and ground-managed metering, merging and spacing.

- Within the optimized metroplex flow, rational trajectories accommodated for aircraft arriving to and departing from small, virtual-tower airports in Instrument Meteorological Conditions and transiting through high-density metroplex airspace.

\section{Gate-to-Gate Scenario}

This section describes a notional gate-to-gate TBO scenario, showing integrated planning and flow management across phases of flight.

\section{Preflight}

Preflight, the FOC uses CATM tools to develop and submit a 4D trajectory for the flight. The preferred windoptimal 2D route for the flight was not available due to predicted en route weather, and the negotiated trajectory follows several sections of published 2D routes defined by the ANSP as a constraint, deviating to the south of the weather. The FOC submits a Trajectory Option Set for the flight with four alternate trajectories that would be more fuel-optimal, which might be accepted later if the weather does not materialize as predicted. Because there are multiple fronts of convective weather across the country, this 4D trajectory includes three TOAC windows, but the FOC has ensured that the aircraft can meet all three negotiated windows with a reasonably smooth and fuel-efficient 4D trajectory. The first TOAC meters the flight into the dense en route stream at Top of Climb, with a window of plus or minus three minutes of the assigned time. The second TOAC is to manage the flow into an en route Flow Constrained Area caused by convective weather. The third TOAC is to manage the flow into the destination airport, which will be capacity-constrained at the time the flight arrives. The third TOAC is one hour later than the published, scheduled arrival time for the flight.

\section{Surface}

Since the flight is impacted by TOACs, the FOC is notified of an expected window for pushback, so the airplane can be loaded at the appropriate time. Once the airplane is loaded, the ANSP is notified that the aircraft is prepared for on-time departure. Surface operations automation, working in an integrated manner with flow management tools across all phases of flight, creates a $2 \mathrm{D}$ taxi route with timing at key points, which is transmitted to the aircraft via DataComm. The timing for taxi-out is created by working backwards to meet the first TOAC, accounting for other departing and arriving flights, sequencing of aircraft at anticipated taxiway intersections and runway crossings, and maintaining a short queue at the departure runway. The pilot reviews and accepts the taxi information. As the aircraft taxis out, the route and guidance for timing are depicted on the onboard surface map and the Head Up Display (HUD). Following the guidance, the pilot taxis out to the short takeoff queue and takes off within three minutes of the planned Weight Off Wheels time.

\section{Departure}

While the aircraft was waiting in the short takeoff queue, the pilot received a DataComm message to negotiate a minor change in the departure route for the aircraft. The DataComm message contains two new constraints from the ANSP: 1) a D-RNP route that is offset from the Standard Instrument Departure (SID), which the ANSP has established to make room for a D-RNP arrival route offset for a Standard Terminal Arrival Route (STAR) that is impacted by convective weather, and 2) because of the extra time for the stream of departing aircraft on this D-RNP to traverse the distance for the offset SID, the previous TOAC for insertion into the en route stream has been moved to five minutes 
later. The pilot uses the FMS to generate a new 4D trajectory, choosing to meet the second and third TOACs by increasing the en route speed during a portion of the flight. An EPP message with the new trajectory is sent to the ANSP, which is accepted. The aircraft progresses to the front of the takeoff queue and is cleared for takeoff. The climb-out trajectory is an OPC within the constraints of the SID, so the altitudes are tailored to this specific aircraft, which is a new "green" low-emissions aircraft. On initial climb-out, the aircraft transmits an EPP message to the ground-based automation that provides the ANSP with the planned climb rate at given times for that aircraft. This allows the ANSP to reduce the large altitude window reserved for its departure, enabling an aircraft arriving at a nearby small airport to safely pass above rather than deviating many miles out of the way. The aircraft continues its departure, on target to arrive at the waypoint to enter the en route stream within the TOAC window.

\section{En Route}

As the aircraft is climbing to enter the en route stream, which is along an established airway in high-density airspace, the pilot receives an updated clearance that will enable the aircraft to enter a gap in the stream. The controller has protected that gap in the stream by assigning TOAC or A-IM clearances to the aircraft already in the stream. Once established in the stream, the controller issues a TOAC or A-IM clearance to this aircraft to maintain the stream for the next thirty minutes through capacity-constrained airspace. Once the aircraft has passed into medium-density airspace, the 4D trajectory leaves the established airway system and traverses a wind-optimal route defined by latitude/longitude waypoints at a speed slightly above the best economic speed to make up for the slight departure delay.

Thirty minutes later, the FOC dispatcher predicts that there will be a large gap in the convective weather ahead and contacts the pilot via the Aircraft Communications Addressing and Reporting System (ACARS) to suggest that the pilot pick his way through the gap forming in the weather rather than deviating so far south to go around it. The pilot concurs, and the FOC dispatcher uses his CATM automation tools to negotiate a new 3D route to go through the gap, a new TOAC for entry into the capacity-constrained en route airspace and a new arrival TOAC, which is much closer to the original arrival time. The new arrival TOAC is available to the dispatcher because he is cancelling a shorter flight that was headed for the same airport. The constraints for the updated trajectory are datalinked to the aircraft by the ANSP automation. The pilot uses the FMS to generate the new 4D trajectory, which is sent via an EPP message to the ground automation and it is accepted. The pilot then executes the new trajectory, and three minutes later turns more northward following the new trajectory.

As the aircraft approaches the dynamic gap in the weather, the pilot determines that the current 4D trajectory will slightly miss the gap and be impacted by convective weather. He uses the TASAR tool on his EFB to calculate a new 4D trajectory that misses the convective weather and does not conflict with other nearby traffic that is also cutting through the gap. The pilot uses TASAR to submit the change in his 4D trajectory to the ANSP, which still meets the downstream TOAC constraints. The trajectory is accepted by the ANSP, and the pilot executes it and transmits an EPP message so the ground-based automation has an accurate depiction of the trajectory. The aircraft is still off the published airways system, and is now nearing the waypoint for the third TOAC, metering traffic into the capacityconstrained destination airport.

\section{Arrival}

Fifteen minutes prior to Top of Descent, the pilot receives a DataComm message with an A-IM clearance assigning a target aircraft for spacing to the final approach fix. The message includes the aircraft's route for arrival and transition to approach, altitude constraints, and target speeds for both the ownship and the target aircraft so that distance-to-go for both aircraft can be calculated by onboard automation (using ADS-B position information) to support A-IM operations. The message also includes the planned runway exit and 2D taxi route, so the crew can review them before commencing the descent. The pilot determines that the arrival and A-IM operations are feasible, and uses the FMS to generate a highly accurate 4D trajectory for a CDA that is tailored for this new "green" aircraft and is also a low-noise approach with late deployment of flaps and gear. The pilots transmits an EPP message to ground automation, and the highly accurate 4D trajectory information is then uplinked to another aircraft, which has the ownship as its A-IM target aircraft. After the aircraft is descending on the assigned STAR, a datalink message is received, updating the 4D trajectory for the A-IM target aircraft, which is destined for the same runway but on a different STAR. Convective weather is unexpectedly moving towards the target aircraft's future path on his STAR. The target aircraft has negotiated with the ANSP for a slight deviation within the STAR corridor to avoid the hazardous weather, and the new 4D trajectory for the target aircraft is transmitted via datalink to the ownship. Onboard guidance shows that the A-IM is still feasible with a slight reduction in speed early in the arrival to absorb delay. The pilot follows the onboard A-IM guidance, merges behind the target aircraft at the appropriate spacing on the approach course, and lands. 


\section{Surface}

A few minutes prior to reaching the final approach fix, the aircraft receives a DataComm message with the finalized taxi route and timing, which is reviewed and accepted by the copilot. Just prior to touching down on the runway, the pilot detects a mild pocket of turbulence and extends the landing slightly, touching down at the far end of the touchdown zone, slightly faster than planned. As the aircraft touches down on the runway, the Navigation Display transitions to a surface map, depicting the planned runway exit and taxi route, but the pilot radios the tower that he will be taking a later runway exit. As the aircraft turns on the later runway exit, the aircraft receives a DataComm message with an updated taxi route and timing. The new route is accepted by the crew and uploaded, and the pilot follows display guidance to meet the timing and holds short of an active runway that he must cross. After a departing aircraft passes, the pilot receives clearance to cross the active runway and continues to taxi to the gate arriving only twenty minutes late for the scheduled arrival. Since the airline has access to accurate trajectory information and surface operations through SWIM, the airline has ensured that the gate is available when the aircraft arrives, and the ground crew is ready to immediately begin servicing the late aircraft to prepare for the next leg of the flight.

\section{Concluding Remarks}

NASA is in the process of developing a far-term concept for gate-to-gate TBO. Within the concept, operators and service providers collaboratively negotiate aircraft trajectories, providing agile, optimized, aircraft-specific routing to meet service provider gate-to-gate flow-management constraints, and increasing capacity by smoothly and effectively combining flight-deck-based and ground-based metering, merging, and spacing in a mixed-equipage environment. While the process is only in the concept exploration phase, this paper highlights some initial observations and outlines the architectural framework and some of the implementation options within the concept. Future plans are to complete the definition of an architectural framework for TBO, conduct a series of studies and simulations to refine the concept, develop and demonstrate the key functionality, and work with the FAA and RTCA to facilitate technology transfer and standards development. As we develop the concept, we are striving to build on the avionics and communications infrastructure defined collaboratively by FAA and EUROCONTROL and to define a concept that is complementary to and evolves naturally from FAA near- and mid-term TBO development and implementation efforts underway.

\section{References}

${ }^{1}$ NextGen Joint Planning and Development Office, "Concept of Operations for the Next Generation Air Transportation System," Version 3.2, 2011.

${ }^{2}$ NextGen Joint Planning and Development Office, "JPDO Trajectory-Based Operations (TBO) Study Team Report," 2011

${ }^{3}$ Single European Sky ATM Research Consortium, "SESAR Concept of Operations," DLT-0612-222-01-00, 2007.

${ }^{4}$ Moore, M.D. et al, "High-Speed Mobility through On-Demand Aviation," 2013 Aviation Technology, Integration, and Operations Conference, Los Angeles, CA, August 2013.

${ }^{5}$ Cate, K.T., "Challenges in Achieving Trajectory-Based Operations," $51^{\text {st }}$ AIAA Aerospace Sciences Meeting, Grapevine TX, January 2013.

${ }^{6}$ Besada, J.A., Frontera, G., Crespo, J., Casado, E., López-Leonés, J., “Automated Aircraft Trajectory Prediction Based on Formal Intent-Related Language Processing," IEEE Transactions on Intelligent Transportation Systems, Vol. 14, No. 3, September 2013.

${ }^{7}$ Paglione, M., Bayaktutar, L., McDonald, G., Bronsvoort, J., “Lateral Intent Error's Impact on Aircraft Prediction.” Air Traffic Control Quarterly, Vol. 18(1), p. 29-63, 2010.

${ }^{8}$ Bronsvoort, J., McDonald, G., Paglione, M., Young, C., Boucquey, J., Hochwarth, J., Gallo, E., "Real-Time Trajectory Predictor Calibration through Extended Projected Profile (EPP) Down-Link," ATM R\&D Seminar, Lisbon, Portugal, June 2015.

${ }^{9}$ EUROCONTROL, "User Manual for the Base of Aircraft Data (BADA) Family 4," EUROCONTROL Experimental Centre, 2012.

${ }^{10}$ Bronsvoort, J., McDonald, G., Vilaplana, M., Besada, J., “Two-Stage Approach to integrated Air/Ground Trajectory Prediction," Journal of Guidance, Control, and Dynamics, Vol 37, No. 6, Nov-Dec, 2014.

${ }^{11}$ Butler, R., Geser, A., Maddalon, J., Muñoz, C., "Formal Analysis of Air Traffic Management Systems: The Case of Conflict Resolution and Recovery," Proceedings of Winter Simulation Conference, New Orleans, December 2003.

${ }^{12}$ Carreno, V.A., Gottliebsen, Hanne, Butler, R.W., Kalvala, S., "Formal Modeling and Analysis of a Preliminary Small Aircraft Transportation System (SATS) Concept,” NASA-TM-2004-212999, March 2004.

${ }^{13}$ Hagen, G., Butler, R.W., "Towards a Formal Semantics of Flight Plans and Trajectories," NASA TM-2014-218662, December 2014. 
${ }^{14}$ Ballin, M. G., Wing, D. J., "Traffic Aware Strategic Aircrew Requests (TASAR),” 12th AIAA Aviation Technology, Integration, and Operations Conference, Indianapolis, Indiana, September 2012.

${ }^{15} \mathrm{McNally}, \mathrm{D}$. et al, "Dynamic Weather Routes: Two Years of Operational Testing at American Airlines," Eleventh USA/Europe Air Traffic Management Research and Development Seminar, June 2015, Lisbon, Portugal.

${ }^{16}$ Gong, C., McNally, D., Lee, C.H., "Dynamic Arrival Routes: A Trajectory-Based Weather Avoidance System for Merging Arrivals and Metering," 2015 AIAA Aviation Forum, Dallas, TX, June 2015.

${ }^{17}$ Krozel, J., Mitchell, J.S.B., Polishchuk, V., and Prete, J., "Maximum Flow Rates for Capacity Estimation in Level Flight with Convective Weather Constraints," Air Traffic Control Quarterly, Vol. 15, No. 3, 2007.

${ }^{18}$ Kicinger, R., Khorrami, B., Prete, J., Penny, S., Myers, T., Hackney, H., "Robust Traffic Flow Management: Coevolutionary Approach," 47 $7^{\text {th }}$ AIAA Aerospace Sciences Meeting, Orlando, FL, January 2009.

${ }^{19}$ Nilim, A., Ghaoui, E., "Algorithms for Air Traffic Flow Management under Stochastic Environments," Proceeding of the 2004 American Control Conference, Boston, MA, June-July 2004.

${ }^{20}$ Idris, H., Vivona, R., Penny, S., Krozel, J., and Bilimoria, K., "Operational Concept for Collaborative Traffic Flow Management based on Field Observations," AIAA 5th Aviation Technology, Integration and Operations Conference, Arlington, VA, September 2005.

${ }^{21}$ Chittargi, K. Martin, L., “Time Based Flow Management (TBFM) as a Service-With NextGen SOA,” Integrated Communications, Navigation and Surveillance Conference, Washington, DC, April 2013.

${ }^{22}$ Swenson, H. N., Hoang, T., Engelland, S., Vincent, D., Sanders, T., Sanford, B., Heere, K., "Design and Operational Evaluation of the Traffic Management Advisor at the Fort Worth Air Route Traffic Control Center," Proceedings of the 1st USA/Europe Air Traffic Management Research and Development Seminar, Saclay, France, June 1997.

${ }^{23}$ Baxley, B., Swenson, H., Prevot, T., Callantine, T., "NASA's ATM Technology Demonstration-1: Integrated Concept of Arrival Operations," 31st Digital Avionics Systems Conference, Williamsburg, VA, October 2012.

${ }^{24}$ Baxley, B., Johnson, W.C., Swenson, H.N., Robinson, J.E., Prevot, T., Callantine, T.J., Scardina, J., Greene, M., "Air Traffic Management Technology Demonstration-1 Concept of Operations (ATD-1 ConOps), Version 2.0," NASA TM-2013218040, Sep. 2013.

${ }^{25}$ U.S. Department of Transportation Office of Inspector General, "FAA Has Not Effectively Deployed Controller Automation Tools That Optimize Benefits of Performance-Based Navigation," Audit Report AV-2015-081, August, 2015.

${ }^{26}$ EUROCONTROL, “Arrival Manager: Implementation Guidelines and Lessons Learned," December 2010.

${ }^{27}$ McNally, David, et al, "Operational Evaluation of Dynamic Weather Routes at American Airlines," Tenth USA/Europe Air Traffic Management Research and Development Seminar, 2013.

${ }^{28}$ Wichman, K. D., Carlsson, G., and Lindberg, G. V., "Flight Trials 'Runway-to-Runway' Required Time of Arrival Evaluations for Time-Based ATM Environment," 20th Digital Avionics Systems Conference, Daytona Beach, FL, 2001.

${ }^{29}$ Wichman, K. D., J. K., Bleeker, O. F., and Rademaker, R. M., "Flight Validations of Downlinked Flight Management System 4D Trajectory,” 26th Digital Avionics Systems Conference, Dallas, TX, 2007.

${ }^{30}$ Klooster, J. K., Wichman, K. D., and Bleeker, "4D Trajectory and Time of Arrival Control to Enable Continuous Descent Arrivals," AIAA Guidance, Navigation, and Control Conference, Honolulu, HI, 2008.

${ }^{31}$ Klooster, J., DelAmo, A., and Manzi, P., "Controlled Time of Arrival Flight Trials—Results and Analysis,” Eighth USA/Europe Air Traffic Management Research and Development Seminar, Napa, CA, 2009.

${ }^{32}$ Wynnyk, C., Balakrishna, M., MacWilliams, P., and Becher, T., "2011 Trajectory Based Operations Flight Trials," Tenth USA/Europe Air Traffic Management Research and Development Seminar, Chicago, IL, 2013.

${ }^{33}$ Mutuel, L.H., Neri, P., and Paricaud, E., "Initial 4D Trajectory Management Concept Evaluation," Tenth USA/Europe Air Traffic Management Research and Development Seminar, Chicago, IL, 2013. 